Psychother Psychosom 2014;83:252-254

DOI: $10.1159 / 000358526$

\title{
Social Exclusion Leads to Divergent Changes of Oxytocin Levels in Borderline Patients and Healthy Subjects
}

\author{
Andrea Jobst ${ }^{\mathrm{a}}$, Anna Albert ${ }^{\mathrm{a}}$, Christine Bauriedl-Schmidt ${ }^{\mathrm{a}}$, \\ Maria Christine Mauer, ${ }^{\mathrm{a}, \mathrm{c}}$, Babette Renneberg ${ }^{\mathrm{b}}$, Anna Buchheim ${ }^{\mathrm{c}}$, \\ Lena Sabass a , Peter Falkai ${ }^{\mathrm{a}}$, Peter Zilla , Frank Padberg ${ }^{\mathrm{a}}$

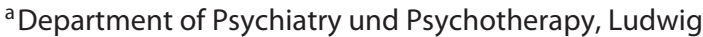 \\ Maximilian University, Munich, and ${ }^{\mathrm{b}}$ Department of Psychology, \\ Free University of Berlin, Berlin, Germany; ${ }^{c}$ Department of \\ Psychology, Clinical Psychology, University of Innsbruck, \\ Innsbruck, Austria
}

Besides affective instability and identity diffusion, borderline personality disorder (BPD) patients show impaired interpersonal functionality [1]. Real or perceived loss, feeling isolated and rejected often evoke impulsive, self-injurious, and suicidal behavior and affective instability in BPD patients [2]. Recently, altered oxytocin regulation has been suggested to be one mechanism underlying such interpersonal dysfunctions in BPD, i.e. reduced plasma oxytocin levels were found in BPD, which were negatively correlated with a history of childhood trauma [3]. To directly address the hypothesis of an altered oxytocin regulation in BPD we exposed 22 women with BPD and 21 healthy controls matched for gender, age and education to a social exclusion (ostracism) situation. Feeling rejected and isolated from others can be experimentally simulated using the Cyberball paradigm, a virtual ball-tossing game during which participants are excluded by the other players [4]. Using this paradigm, higher scores of negative emotions were observed in BPD patients after exclusion compared to healthy controls and patients felt more readily excluded even when they were not [5].

All patients met DSM-IV criteria of BPD (SCID-II interview). Most BPD patients received pharmacological treatment, i.e. antidepressants $(n=15)$, second-generation antipsychotics $(n=14)$, or mood stabilizers $(n=8)$. All participants played Cyberball in a standardized experimental setting. Emotional reactions were assessed by self-rating questionnaires before and after Cyberball and oxytocin and cortisol (to assess stress related effects) plasma levels were measured at baseline (t0) and $5(\mathrm{t} 1), 15(\mathrm{t} 2)$, and $40 \mathrm{~min}(\mathrm{t} 3)$ after Cyberball (fig. 1a). Tubes were prepared with aprotinin (500 $\mathrm{IU} / \mathrm{ml}$ ) and afterwards centrifuged at $1,600 \mathrm{~g}$ for $15 \mathrm{~min}$ and stored at $-80^{\circ} \mathrm{C}$ prior to the biochemical analyses. Serum cortisol levels were determined with an immunoassay analyzer (Elecsys Cortisol Test; Roche Diagnostics, Mannheim, Germany). Oxytocin was assessed by a commercially available ELISA kit (Enzo Life Sciences, Germany). Special attention was paid to the menstrual cycle. Par-

\section{KARGER}

E-Mail karger@karger.com

www.karger.com/pps ticipants taking hormonal contraception were measured between the $3 \mathrm{rd}$ and 18th day of the intake period, and women not taking hormonal contraception were measured within the follicular phase, i.e. between the 5 th and 12 th day of the menstrual cycle. All measurements took place in the morning between 8 and 11 a.m. to control for the circadian change of hormones. As psychological measures, the Childhood Trauma Questionnaire (CTQ) [6], the Rejection Sensitivity Questionnaire (RSQ) [7], a 14-item emotion scale [8] and the Needs-Threat Questionnaire (NTQ) [9] were used. In addition, we asked the participants to assess aversive inner tension, expressed as a percentage of maximal tension.

For oxytocin, a significant time $\times$ group interaction was observed at $\mathrm{t} 0$ and $\mathrm{t} 1\left(\mathrm{~F}=4.957\right.$; d.f. $\left.=1 ; \mathrm{p}=0.032^{*}\right)$. BPD subjects showed a reduction of oxytocin plasma levels following social exclusion compared to healthy subjects (fig. 1b). There were no significant effects for time $(\mathrm{F}=0.271$; d.f. $=1 ; \mathrm{p}=0.605)$ or group $(\mathrm{F}=0.987$; d.f. $=1 ; \mathrm{p}=0.327)$. The RM ANOVA over all four time points did not show significant results (time: $\mathrm{F}=0.533$; d.f. $=2.427$; $\mathrm{p}=0.623$; time $\times$ group: $\mathrm{F}=1.467 ;$ d.f. $=2.427 ; \mathrm{p}=0.234$, group: $\mathrm{F}=0.693$; d.f. $=1 ; \mathrm{p}=0.411$ ). Independent sample $\mathrm{t}$ test revealed a different direction of change in oxytocin levels from t0 to t 1 between healthy controls and BPD patients $(\mathrm{t}=2.227$; d.f. $=37 ; \mathrm{p}=$ $\left.0.032^{*}\right)$. Baseline oxytocin peripheral levels were not correlated with age, severity of clinical symptoms, or most measures of the emotional reaction, or related to the menstrual cycle. In the BPD group, we found a negative association between physical and emotional abuse during childhood (CTQ) and return of oxytocin levels to baseline. The higher the level of emotional $(\mathrm{r}=-0.449 ; \mathrm{p}=$ $\left.0.042^{*}\right)$ and physical $\left(r=-0.465 ; p=0.039^{*}\right)$ abuse was, the smaller the change of oxytocin became. Cortisol levels showed no increase following social exclusion and no significant difference between groups (fig. 1c), but a decrease due to circadian decline (RM ANOVA: significant effect for time: $\mathrm{F}=34.301$; d.f. $=1.392 ; \mathrm{p}<$ 0.001 ; but not for time $\times$ group interaction: $\mathrm{F}=0.882$; d.f. $=1.392$; $\mathrm{p}=0.385$ or for group difference: $\mathrm{F}=0.025$; d.f. $=1 ; \mathrm{p}=0.875$ ). There was no correlation between oxytocin and cortisol levels.

Inner tension significantly differed between groups with higher inner tension in BPD (RM ANOVA: $\mathrm{F}=15.716$; d.f. $=1 ; \mathrm{p}<0.001^{*}$ ), but playing Cyberball did not significantly change the amount of inner tension in either group (no time interaction: $\mathrm{F}=0.026$; d.f. $=$ $1 ; \mathrm{p}=0.873$ and no time $\times$ group interaction: $\mathrm{F}=0.104 ;$ d.f. $=1$; $\mathrm{p}=0.749$ ). All participants reported disregard and exclusion when answering the NTQ index questions (mean ratings $>3$ ). BPD patients had a significantly higher rejection sensitivity (RSQ: $\mathrm{t}=$ -8.474 ; d.f. $\left.=27 ; \mathrm{p}=<0.001^{*}\right)$ and felt more readily excluded than controls (NTQ: $\mathrm{t}=-2.359$; d.f. $=35 ; \mathrm{p}=0.024^{*}$ ), even though both the BPD and control groups judged ball ownership realistically and felt disregarded to a similar extent. However, the exclusion paradigm had a significantly higher aversive impact on BPD patients than on controls (NTQ: aversive impact factor: $\mathrm{t}=-2.815$; d.f. $=$ $\left.30.751 ; \mathrm{p}=0.008^{*}\right)$. After social exclusion, BPD patients remained in more pronounced negative emotional states: they experienced 


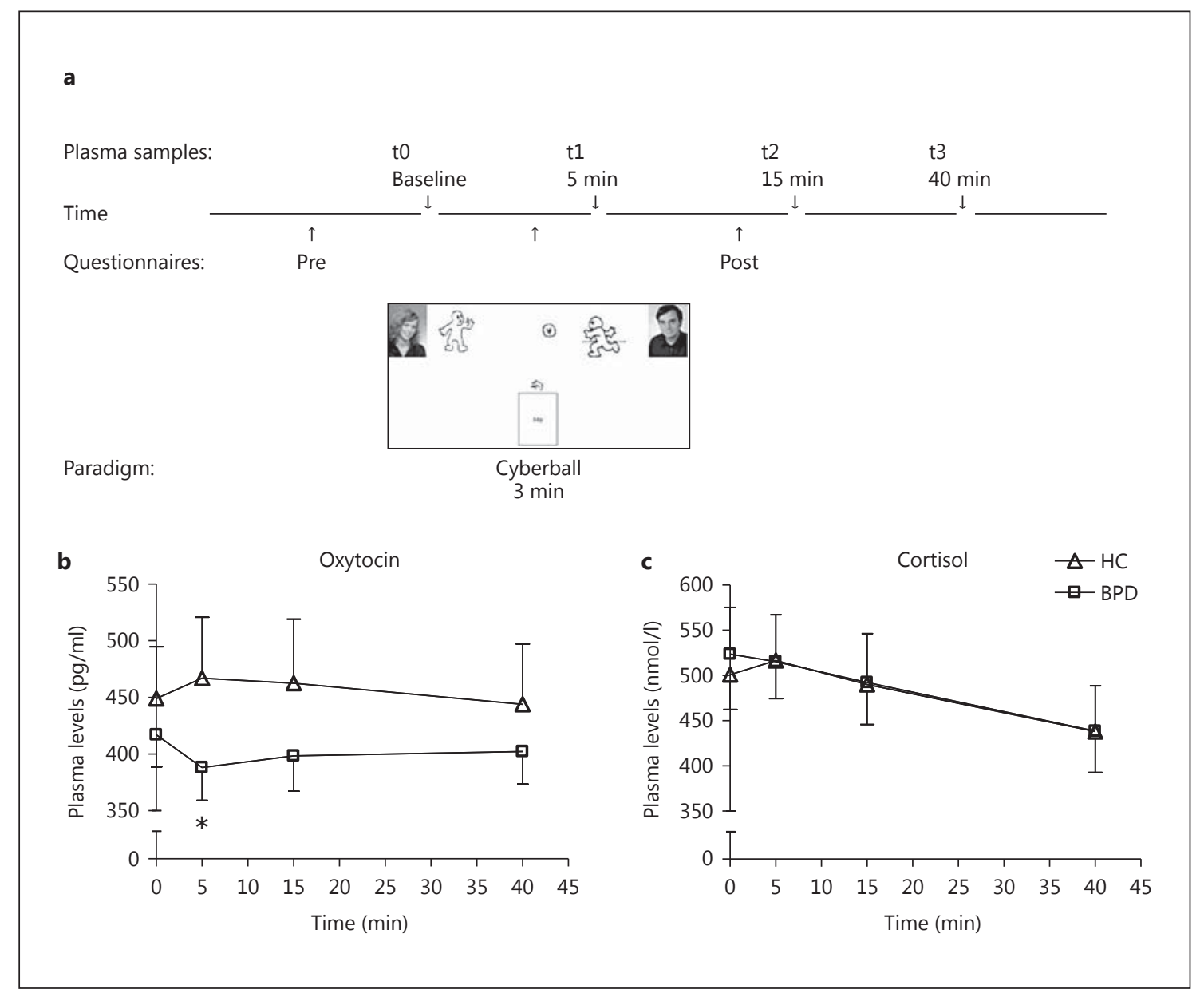

Fig. 1. a Experimental design: oxytocin and cortisol levels were measured at four time points before and after Cyberball. b Peripheral oxytocin levels. c Cortisol levels. A repeated measurements ANOVA for baseline and $\mathrm{t} 1$ showed a significant difference for the development over time between BPD patients and healthy controls (HC, $\mathrm{F}=4.957$; d.f. $\left.=1 ; \mathrm{p}=0.032^{*}\right)$. Cortisol decreased significantly over time in both groups $(\mathrm{F}=34.301 ; \mathrm{d} . \mathrm{f} .=1.392$; $\left.\mathrm{p}=0.001^{*}\right)$, as expected according to its circadian change.

more negative emotions during the game (negative mood: $\mathrm{t}=$ -2.637 ; d.f. $=36 ; p=0.012 *$, and their needs were more threatened during the exclusion paradigm than were those of controls (needs: $\mathrm{t}=3.595$; d.f. $\left.=36 ; \mathrm{p}=0.001^{*}\right)$. Moreover they showed an increase in negative emotions focused on others [RM-ANOVAs: significant time $\left(\mathrm{F}=6.065\right.$; d.f. $\left.=1 ; \mathrm{p}=0.019^{*}\right)$ and group effects $(\mathrm{F}=14.474$; d.f. $\left.=1 ; \mathrm{p}=0.001^{*}\right)$ ], in particular anger $(\mathrm{t}=-2.251 ;$ d.f. $=18 ; \mathrm{p}=$ $\left.0.037^{*}\right)$ and contempt $\left(\mathrm{t}=-2.480\right.$; d.f. $\left.=18 ; \mathrm{p}=0.023^{*}\right)$, whereas this was not the case in controls. In this respect, our results basically replicate a previous study by Renneberg et al. [5].

The main finding was the differential regulation of oxytocin plasma levels in BPD patients and healthy subjects after social exclusion, whereas cortisol levels did not differ between BPD patients and controls. Our findings support the notion that an altered regulation of the oxytocin system may underlay BPD patients' deficits in repairing broken cooperation [10]. Recently, reduced plasma oxytocin levels were found in female BPD patients compared to controls [3]. In contrast, we did not observe a significant difference in baseline oxytocin levels between BPD patients and controls; however, this may be due to the small sample size. In summary, our pilot study shows a reduction of oxytocin plasma levels after social exclusion in BPD patients compared to healthy controls. To our knowledge, this is the first study investigating oxytocin plasma levels during a social exclusion paradigm.

\section{Acknowledgments}

This study is part of A.A.'s MD thesis at the Faculty of Medicine of the Ludwig Maximilian University, Munich (in preparation). The study was supported by the FöFoLe Program of the Faculty of Medicine of the Ludwig Maximilian University, Munich (grant to A.J.).

We thank the clinicians, psychologists, and students, who provided support for the study. 


\section{Disclosure Statement}

F.P. has received grants and research support from Brainsway Inc., Israel, and neuroConn $\mathrm{GmbH}$, Ilmenau, Germany. Until 2010, P.F. received grants and research support from Astra Zeneca, speakers honoraria from Astra Zeneca, BMS, Essex, GSK, JanssenCilag, and Lundbeck, and was a speaker or member of the advisory boards of Janssen-Cilag, Astra Zeneca, Lilly, and Lundbeck.

\section{References}

1 Bohus M, Kröger C: Psychopathology and psychotherapy of borderline personality disorder: state of the art (in German). Nervenarzt 2011;82: $16-24$.

-2 Stiglmayr CE, Grathwol T, Linehan MM, Ihorst G, Fahrenberg J, Bohus M: Aversive tension in patients with borderline personality disorder: a computer-based controlled field study. Acta Psychiatr Scand 2005;111: 372-379.

-3 Bertsch K, Schmidinger I, Neumann ID, Herpertz SC: Reduced plasma oxytocin levels in female patients with borderline personality disorder. Horm Behav 2012;63:424-429.
4 Williams KD, Jarvis B, Cyberball: A program for use in research on interpersonal ostracism and acceptance. Behav Res Methods 2006;38:174180.

5 Renneberg B, Herm K, Hahn A, Staebler K, Lammers CH, Roepke S: Perception of social participation in borderline personality disorder. Clin Psychol Psychother 2012;19:473-480.

6 Klinitzke G, Romppel M, Häuser W, Brähler E, Glaesmer H: The German Version of the Childhood Trauma Questionnaire (CTQ): psychometric characteristics in a representative sample of the general population (in German). Psychother Psychosom Med Psychol 2012;62:47-51.

-7 Staebler K, Helbing E, Rosenbach C, Renneberg B: Rejection sensitivity and borderline personality disorder. Clin Psychol Psychother 2011;18: 275-283.

8 Gross JJ, Levenson RW: Emotion elicitation using films. Cognition Emotion 1995;9:87-108.

9 Williams KD, Cheung CK, Choi W: Cyberostracism: effects of being ignored over the Internet. J Pers Soc Psychol 2000;79:748-762.

10 King-Casas B, Sharp C, Lomax-Bream L, Lohrenz T, Fonagy P, Montague PR: The rupture and repair of cooperation in borderline personality disorder. Science 2008;321:806-810. 